\title{
Fronteiras: Revista Catarinense de História
}

Programa de Pós-Graduação em História da UFFS e ANPUH-Seção SC (Gestão 2018-2020).

Editora: Samira Peruchi Moretto

Conselho Editorial: Alessandra Izabel de Carvalho - Universidade Estadual de Ponta Grossa - UEPG (Brasil); Antonio MartínezArboleda - University of Leeds (Reino Unido); Ben Cowan - University of California San Diego - UCSD (EUA); Benito Bisso Schmidt Universidade Federal do Rio Grande do Sul - UFRGS (Brasil); Ely Bergo de Carvalho - Universidade Federal de Minas Gerais - UFMG (Brasil); Joana Maria Pedro - Universidade Federal de Santa Catarina - UFSC (Brasil); Jose Miguel de Toro Vial - Universidade Católica da Santíssima Conceição - UCSC (Chile); Leandro Duarte Rust Universidade Federal do Mato Grosso - UFMT (Brasil); Marcelo Cândido da Silva - Universidade de São Paulo - USP (Brasil); Patricia Melo Sampaio - Universidade Federal do Amazonas - UFAM (Brasil); Sandro Dutra e Silva - Universidade Estadual de Goiás - UEG (Brasil); Fernando Cesar Sossai, Universidade da Região de Joinville UNIVILLE (Brasil); Michele Gonçalves Cardoso - Universidade do Extremo Sul Catarinense - UNESC (Brasil);

Conselho Consultivo: Alessandra Izabel de Carvalho (UEPG), Antonio Martínez-Arboleda (University of Leeds, Reino Unido), Ben Cowan (UCSD, EUA), Benito Bisso Schmidt (UFRGS), Ely Bergo de Carvalho (UFMG), Joana Maria Pedro (UFSC), Jose Miguel de Toro Vial (UCSC, Chile), Leandro Duarte Rust (UFMT), Marcelo Cândido da Silva (USP), Patricia Melo Sampaio (UFAM) e Sandro Dutra e Silva (UEG). PORTARIA N 429/GR/UFFS/2019.

Organização do Dossiê Infâncias, Direitos e Vulnerabilidades: Aline Fátima Lazarotto (Unochapecó), Camila Serafim Daminelli (IFES), Elisangela da Silva Machieski (UENP).

Pareceristas deste número: Ailton José Morelli (UEM), Ana Carla Vagliati (Unicesumar), Anderson Marcelo Schmitt (UFSC), Assis da Costa Oliveira (UFPA), Cleonice Elias da Silva (UENP), Consuelena Lopes Leitão (UFAM), Daniel Alves Boeira (Acaps/SC), Eduardo Silveira Netto Nunes (UniSant'Anna), Humberto da Silva Miranda (UFRPE), Ismael Gonçalves Alves (UNESC), Jorge Luiz Zaluski 
(Unicentro), José Carlos da Silva Cardoso (FURG), José dos Santos Costa Júnior (IFRN), Marcelle Jacinto da Silva (UFC), Maria Nilvane Fernandes (UFAM), Olga Brites (PUC/SP), Ricardo Peres da Costa (POIÉSIS), Silvia Maria Fávero Arend (UDESC), Vera Lúcia Braga de Moura (SED/PE).

Preparação dos originais para publicação do número 38: Samira Peruchi Moretto e Michely Cristina Ribeiro

Imagem da capa do número 38: IP1629 - Acervo Irmandade do Divino Espírito Santo Florianópolis-SC. Digitalizado por: UDESC/FAED-IDCH.

Fronteiras: Revista Catarinense de História [Associação Nacional de História - Seção Santa Catarina e PPGH/UFFS]. Chapecó, julho de 2018. [on-line]. Semestral. A publicação segue o Acordo Ortográfico da Língua Portuguesa aprovado pelos países lusófonos em dezembro de 1990, com início de vigência no Brasil a partir de $1^{\circ}$. de janeiro de 2009.

Fronteiras: Revista Catarinense de História é uma publicação anual editada pelo Programa de Pós-graduação em História da Universidade Federal de Santa Catarina (PPGH/UFFS) e Associação Nacional de História - Seção Santa Catarina (ANPUH-Seção SC), voltada para a veiculação de produção atual de caráter histórico. Os autores são responsáveis pelas opiniões expressas em seus trabalhos, bem como pela revisão ortográfica, das normas da ABNT.

Endereço para correspondência: Av. Fernando Machado, $108 \mathrm{E}$, Centro, Chapecó, SC - Brasil Caixa Postal 181 - CEP 89802-112

E-mail: fronteirasanpuhsc@gmail.com;

Homepage: https://periodicos.uffs.edu.br/index.php/FRCH/index

Fronteiras: Revista Catarinense de História. Florianópolis, n. 38 p. 01 a 298. Dezembro de 2021. 